\title{
S-1 Plus Leucovorin and Oxaliplatin in Combination with Lentinan as First-line Therapy in Patients with Metastatic Gastric Cancer
}

\author{
Kenji Ina ${ }^{1^{*}}$, Ryuichi Furuta ${ }^{2}$, Megumi Kabeya ${ }^{3}$, Takashi Yoshida ${ }^{4}$, Takae Kataoka ${ }^{4}$ and \\ Satoshi Kayukawa ${ }^{4}$
}

${ }^{1}$ Department of Psychosomatic Medicine, Nagoya Memorial Hospital, Japan

${ }^{2}$ Department of Medical Oncology, Nagoya Memorial Hospital, Japan

${ }^{3}$ Department of Hospital Pharmacy, Nagoya Memorial Hospital, Japan

${ }^{4}$ Department of Clinical Oncology, Nagoya Memorial Hospital, Japan

\begin{abstract}
Background: Metastatic gastric cancer has a poor prognosis, despite recent therapeutic improvements. The phase 3 SOLAR study confirmed better efficacy of S-1, oxaliplatin, and leucovorin (SOL) than S-1 plus cisplatin in advanced gastric cancer. Lentinan, $\beta-(1,3)$-glucan purified from Shiitake mushrooms, has been reported to improve the overall survival of cancer patients receiving chemotherapy. We conducted a preliminary study of SOL combined with lentinan during these 4 years.

Methods: The clinical study was approved by the ethics committee of Nagoya Memorial Hospital in 2016. After explaining the protocol of this study, patients with recurrent or unresectable gastric cancer were enrolled, if they had the intention to participate. Medical records were retrospectively reviewed to determine the objective response rate (ORR), disease control rate, overall survival, and adverse effects.

Results: Twelve patients (age: 59-81 years; sex: 9 men, 3 women) with metastatic gastric cancer (liver: 3 , lung: 2 , peritoneal: 12, ascites: 9) were treated with SOL in combination with lentinan as the first-line regimen. The cycles ranged from 4 to 15. The ORR and disease control rates were 58.3\% (complete response [CR], 1; partial response, 6) and $91.7 \%$, respectively. One patient with CR survived for $>23$ months after the initiation of chemotherapy. Concerning adverse events, peripheral neuropathy was the most common event observed in all patients. However, there were no severe side effects, such as febrile neutropenia and diarrhea.

Conclusions: SOL combined with lentinan can be a promising option for the treatment of far advanced metastatic gastric cancer.
\end{abstract}

Keywords: Gastric cancer, SOL, Lentinan.

\section{INTRODUCTION}

Gastric cancer remains the fifth most common malignancy and the fourth leading cause of cancerrelated mortality worldwide [1]. Chemotherapy is recommended for patients with metastatic gastric cancer for palliative purposes, considering the possibility of improving the overall survival (OS) using chemotherapy compared with using supportive care [2, 3]. Despite the development of chemotherapeutic and biological agents, the OS of patients with unresectable or recurrent gastric cancer remains less than 2 years $[4,5]$. Lentinan, the backbone of $\beta-(1,3)$-glucan with $\beta$ $(1,6)$ branches, is an active ingredient purified from Shiitake mushrooms [6]. This $\beta$-glucan has been reported to improve the OS of cancer patients receiving chemotherapy [7,8], though inconsistent results have been presented [9]. Cancer cells express many inhibitory signaling proteins that enable their survival in

*Address correspondence to this author at the Department of Psychosomatic Medicine, Nagoya Memorial Hospital, 4-305 Hirabari, Tenpaku-ku, Nagoya 468-8520, Japan; Tel: +052-804-1111; Fax: +052-803-8830;

E-mail: kina@hospy.or.jp the host. Such immune evasion is essential for cancer development, progression, and chemoresistance [10]. One such inhibitory molecule is programmed cell death ligand 1 (PD-L1), which engages programmed cell death receptor 1 (PD-1) expressed by activated-T cells and subsequently triggers inhibitory signaling pathways downstream the T-cell antigen receptors. This protein can shield tumor cells and protect them from lysis via cytotoxic $T$ lymphocytes, suggesting that upregulation of PD-L1 in cancer cells might mediate immune escape [11]. We previously demonstrated that treatment with either cisplatin or oxaliplatin dose-dependently enhanced PD-L1 mRNA and protein expression in gastric cancer cells, leading to the resistance to platinum-based chemotherapy. On the other hand, lentinan treatment inhibited the platinum-induced increase in the expression of PD-L1 and mitogenactivated protein kinase [12], which implicated that lentinan could restore the chemosensitivity of cells through downregulating PD-L1 expression. This action of lentinan might explain the mechanism of complete tumor clearance in patients with metastatic gastric cancer receiving chemo-immunotherapy [13]. 
Several chemotherapeutic agents, including fluorouracil, platinum, and taxanes, are active in advanced gastric cancer. In Japan, combination therapy using the oral fluoropyrimidine anticancer agent S-1 and cisplatin is the standard therapy for metastatic gastric cancer [14]. Recent studies revealed that $\mathrm{S}-1$, oxaliplatin, and leucovorin (SOL) was more active than S-1 plus cisplatin in the Asian patients with advanced gastric cancer $[5,15]$.

Therefore, we conducted a preliminary study of SOL combined with lentinan for patients with unresectable or recurrent gastric cancer.

\section{PATIENTS AND METHODS}

The clinical study was approved by the ethics committee of Nagoya Memorial Hospital in 2016. After explaining the protocol of this study, patients with recurrent or unresectable gastric cancer were enrolled and provided their informed consent for participating in this study. Medical records were retrospectively reviewed to determine the objective response rate (ORR), disease control rate, OS, and adverse effects. The dose of $\mathrm{S}-1$ for each administration was determined according to the body surface area (BSA) as follows: $40 \mathrm{mg}$ for BSA $<1.25 \mathrm{~m}^{2}, 50 \mathrm{mg}$ for BSA $1.25-1.50 \mathrm{~m}^{2}$, and $60 \mathrm{mg}$ for $B S A \geq 1.5 \mathrm{~m}^{2}$. S-1 was orally administered along with $25 \mathrm{mg}$ of leucovorin twice daily for 7 days, and oxaliplatin $\left(85 \mathrm{mg} / \mathrm{m}^{2}\right)$ and 2 $\mathrm{mg}$ of lentinan were administered intravenously on day 1 , every 2 weeks.

The objective response to chemotherapy was evaluated using the criteria proposed by the Japanese
Research Society for Gastric Cancer for the Primary Lesion [16] and using the Response Evaluation Criteria in Solid Tumors (RECIST version 1.1) [17] for metastatic lesions. The disappearance of all cancer evidence for at least 4 weeks was defined as complete response (CR). According to the RECIST, at least a $30 \%$ decrease in the sum of the diameters of target lesions was defined as partial response (PR). The development of a new lesion or at least a $20 \%$ increase in the sum of the diameters of target lesions was defined as progressive disease (PD). Patients who did not satisfy the criteria for any of these categories were considered to have stable disease (SD). Disease control was defined as CR, PR, or SD. The National Cancer Institute Common Toxicity Criteria version 4.0 was used to evaluate adverse effects. Doses were adjusted at the initiation of subsequent cycles if severe toxicity (grade 3-4) was present. If neurotoxicity progressed to grade 2, oxaliplatin was discontinued.

OS was calculated from the start of chemoimmunotherapy until death or the most recent follow-up date. The Kaplan-Meier method was used to plot the OS curves, and OS rates were compared between patients showing objective responses (CR and PR) and those without objective responses (SD and PD) using the log-rank test [18].

\section{RESULTS}

Twelve patients (age: 59-81 years; sex: 9 men, 3 women) with metastatic gastric cancer (liver: 3 , lung: 2 , peritoneal: 12, ascites: 9) were treated with SOL combined with lentinan as a first-line regimen (Table 1). The performance status was 0 in two patients, 1 in nine

Table 1: List of Patients with Metastatic or Recurrent Gastric Cancer Receiving S-1, Oxaliplatin, and Leucovorin Combined with Lentinan

\begin{tabular}{|c|c|c|c|c|c|c|c|c|c|c|c|}
\hline Case No & Age & Sex & $\mathbf{T}$ & $\mathbf{N}$ & M & Liver & Lung & Peritoneum & Ascites & Response & PS \\
\hline 1 & 81 & $M$ & $\mathrm{~T} 4$ & N3 & M1 & - & - & + & + & PR & 1 \\
\hline 2 & 61 & $\mathrm{~F}$ & T4 & N3 & M1 & - & - & + & + & SD & 1 \\
\hline 3 & 69 & $\mathrm{~F}$ & $\mathrm{~T} 4$ & N3 & M1 & - & - & + & + & PR & 1 \\
\hline 4 & 63 & $M$ & T3 & N3 & M1 & - & - & + & + & PR & 1 \\
\hline 5 & 74 & $M$ & $\mathrm{~T} 4$ & N3 & M1 & + & + & + & + & SD & 1 \\
\hline 6 & 74 & M & $\mathrm{T} 4$ & $\mathrm{~N} 2$ & M1 & - & - & + & + & PD & 1 \\
\hline 7 & 73 & $M$ & \multicolumn{3}{|c|}{ Recurrence } & + & - & + & + & PR & 1 \\
\hline 8 & 80 & $M$ & \multicolumn{3}{|c|}{ Recurrence } & - & - & + & - & PR & 0 \\
\hline 9 & 59 & $M$ & T4 & $N X$ & M1 & - & - & + & - & CR & 1 \\
\hline 10 & 70 & $M$ & $\mathrm{~T} 4$ & N3 & M1 & + & - & + & + & SD & 2 \\
\hline 11 & 72 & $M$ & \multicolumn{3}{|c|}{ Recurrence } & - & + & + & + & SD & 1 \\
\hline 12 & 60 & $\mathrm{~F}$ & \multicolumn{3}{|c|}{ Recurrence } & - & + & + & - & PR & 0 \\
\hline
\end{tabular}

PS: performance status; CR: complete response; PR: partial response; SD: stable disease; PD: progressive disease. 


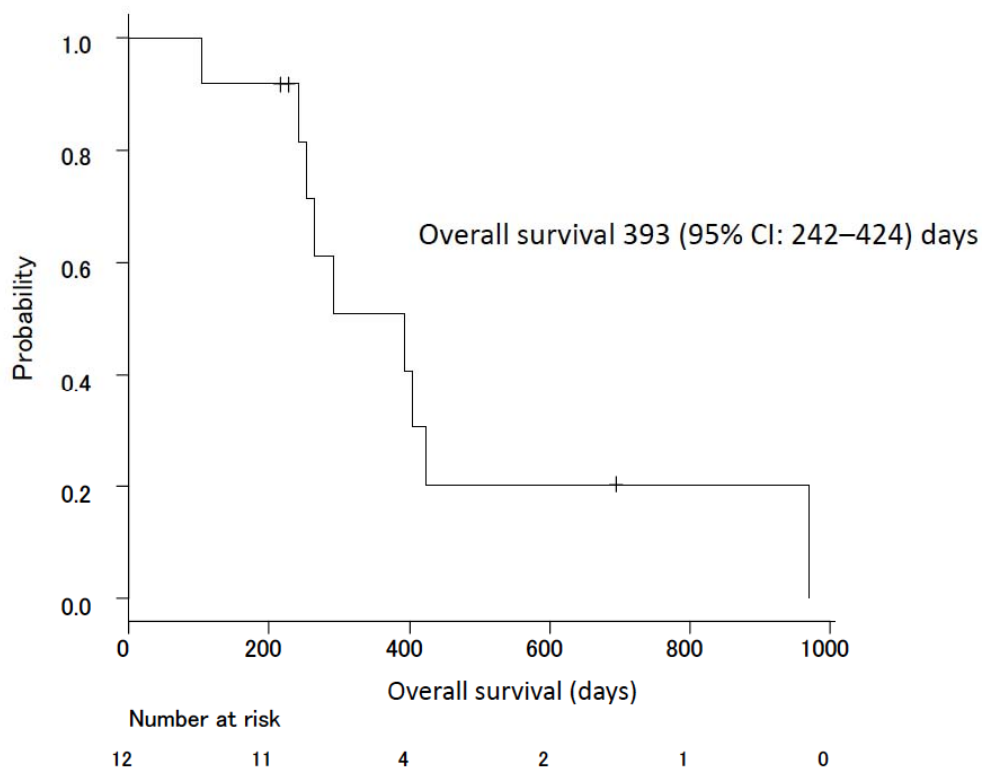

Figure 1: Kaplan-Meier curves of overall survival in 12 patients with metastatic or recurrent gastric cancer receiving S-1, oxaliplatin, and leucovorin combined with lentinan.

patients, and 2 in one patient. The cycles ranged from 4 to 15. The ORR and disease control rates were $58.3 \%$ (CR 1, PR 6) and 91.7\%, respectively. One patient with $\mathrm{CR}$ survived for $>23$ months without any recurrence. The most common grade 1 or 2 adverse events were peripheral neuropathy (grade 2: 6, grade 1: 6), followed by anemia (grade 2: 1 , grade 1: 8), leucopenia (grade 2: 3 , grade 1: 5), neutropenia (grade 2: 7 ), fatigue (grade 2: 3 , grade 1: 3 ), anorexia (grade 2: 4, grade 1: 1), diarrhea (grade 2: 2, grade 1: 1), and thrombocytopenia (grade 2: 1, grade 1: 1). The most common grade 3-4 hematological adverse event was neutropenia (grade 3: 1, grade 4: 1). However, there were no treatment-related deaths. The median OS of 12 patients was 393 days (95\% Cl: 242424 days) (Figure 1), and three patients remain still alive (CR 1, PR 1, SD 1). When comparing the patients showing objective response $(n=7)$ with those with $S D$ or PD $(n=5)$, OS did not significantly differ between the two groups.

\section{CASE REPORT}

A 59-year old man complained of loss of appetite, vomiting, and weight loss (Case 9). Computed tomography (CT) at the time of the first visit revealed severe dilatation of the stomach with wall thickening of the antrum (Figure 2A). After intubation of the nasogastric tube to drain the gastric juice, a scirrhous type of gastric cancer with pyloric stenosis was diagnosed using a gastrointestinal fiberscope (Figure 3A). During the surgical procedure for pyloric stenosis, peritoneal dissemination was detected, and bypass surgery was performed with the diagnosis of T4NXM1. The regimen of SOL plus lentinan was initiated for the treatment of scirrhous type of gastric cancer accompanied by cancerous peritonitis. After nine cycles of chemo-immunotherapy, re-evaluation was performed, which revealed a good extension of the gastric lumen as well as the loss of ulcerative lesions (Figure 3B). Pathological examination of the biopsy specimens was negative. Fifteen months after the initiation of SOL therapy combined with lentinan, further improvement in both $\mathrm{CT}$ and endoscopic findings was observed (Figures 2B, 3C). Consequently, the patient achieved $\mathrm{CR}$, and because of the strong hope of this patient, chemo-immunotherapy was discontinued. He remains alive without any symptoms 8 months after the cessation of treatment.

\section{DISCUSSION}

Recently, SOL has been reported to be the new first-line chemotherapy option for advanced gastric cancer in Asian populations based on the improved response rate and OS, compared with those of S-1 plus cisplatin [5]. This study also supported the promising efficacy and safety of this regimen for advanced gastric cancer. However, the efficacy of chemotherapeutic agents is usually limited owing to patients' resistance to conventional treatments. Such resistance to chemotherapy may be associated with the upregulation of PD-L1 caused by platinum-based treatment $[19,20]$. 
A

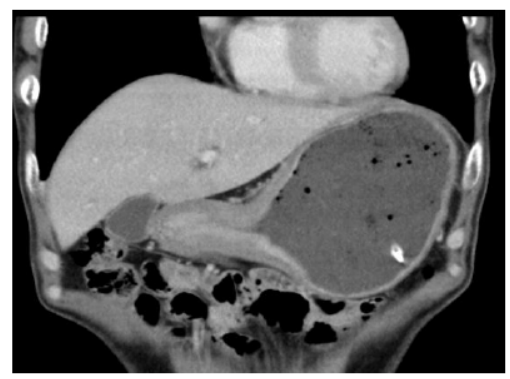

B

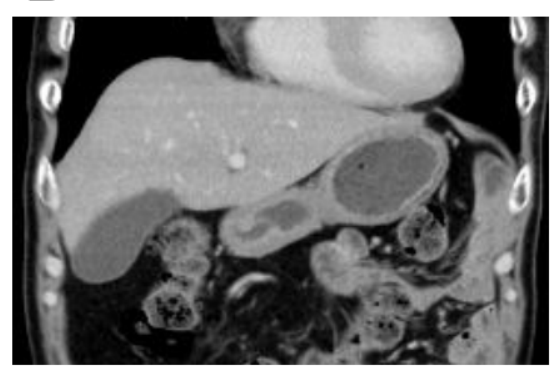

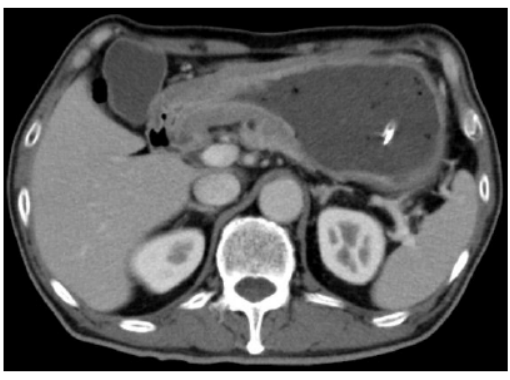

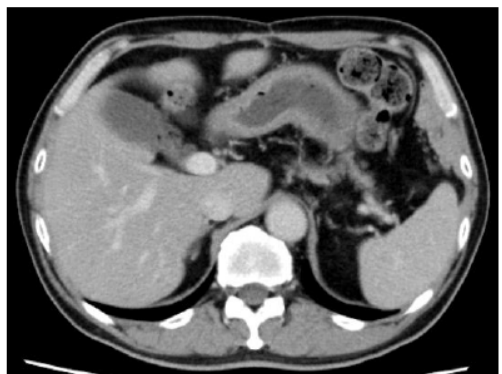

Figure 2: Computed tomography findings of Case 9.

A. Before chemotherapy.

B. Fifteen months after the initiation of chemotherapy.

A
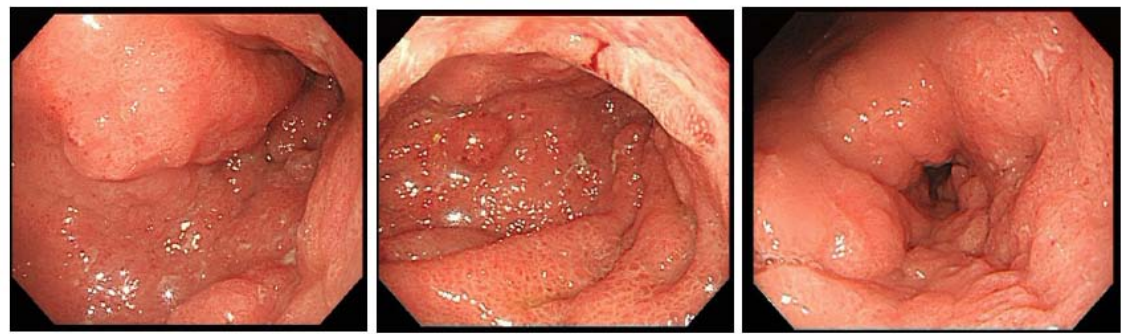

B
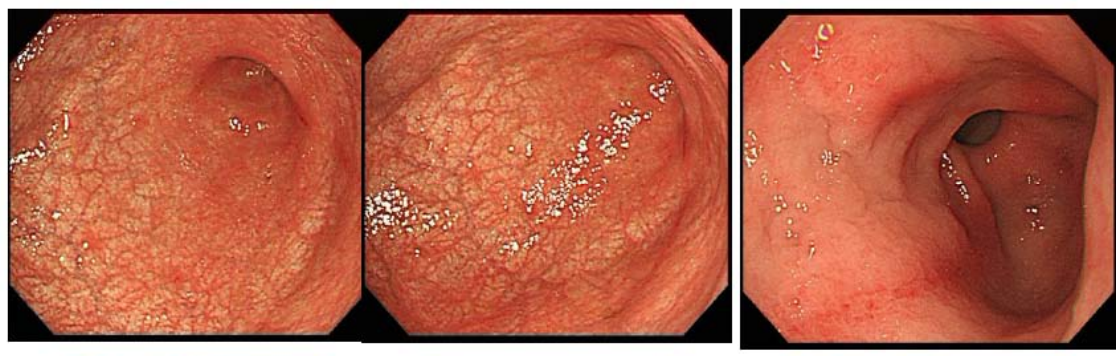

C

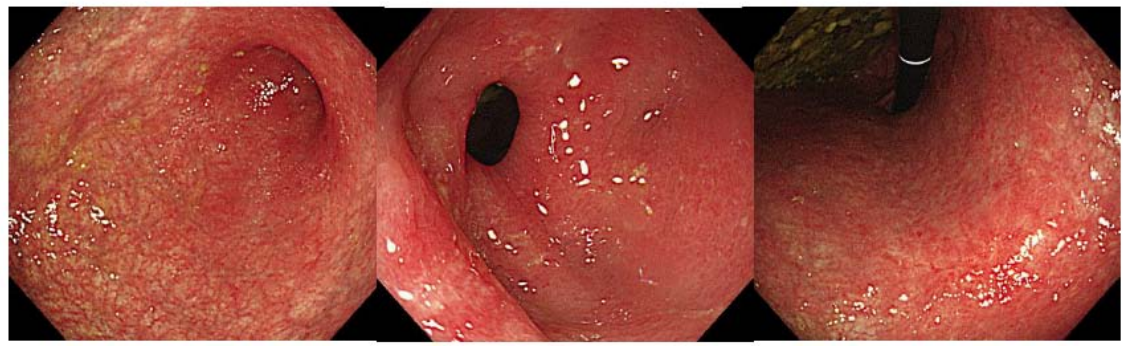

Figure 3: Endoscopic findings of Case 9.
A. Before chemotherapy.
B. Five months after the initiation of chemotherapy.
C. Fifteen months after the initiation of chemotherapy. 
$\beta$-glucans are well-established natural immune modulators with significant anticancer properties [21, 22]. Among the glucans, lentinan is especially remarkable for its immunomodulating [23] and anticancer activities [24]. Additional treatment with lentinan has been reported to prolong the survival of patients with cancer when compared with chemotherapy alone [7, 8]. Lentinan might restore sensitivity to conventional chemotherapy by downregulating PD-L1 expression induced by platinum [12], which may contribute to tumor clearance by T-cell mediated immune responses of the host.

Though $C R$ is rarely attained in patients with metastatic gastric cancer receiving systemic chemotherapy [25], we experienced one such case among the 12 patients enrolled in this study. Chemoimmunotherapy using lentinan might raise the possibility of complete elimination of tumor cells through synergistic effects between lentinan and platinum-based chemotherapy.

\section{CONCLUSION}

SOL combined with lentinan can be a promising option for the treatment of far advanced metastatic gastric cancer owing to its safety and characteristic of enhancing immune responses.

\section{COMPETING INTERESTS}

The authors declare no competing interests.

\section{AUTHORS' CONTRIBUTIONS}

$\mathrm{KI}$ wrote the manuscript. $\mathrm{KI}$ and $\mathrm{RF}$ treated metastatic gastric cancer. RF and SK interpreted the radiological examination findings. MK performed the statistical analysis. TY, TK, and SK reviewed the manuscript. All authors have read and approved the final manuscript.

\section{REFERENCES}

International Agency for Research on Cancer. GLOBOCAN 2020: estimated cancer incidence, mortality and prevalence worldwide. Available online: http://globocan.iarc.fr/Default. aspx (accessed on 11 January 2021).

[2] Glimelius B, Ekstrom K, Hoffman K, et al. Randomized comparison between chemotherapy plus best supportive care with best supportive care in advanced gastric cancer. Ann Oncol 1997; 8: 163-8. https://doi.org/10.1023/A:1008243606668

[3] Hohenberger P, Gretshel S. Gastric cancer. Lancet 2003; 362: 305-15

https://doi.org/10.1016/S0140-6736(03)13975-X
[4] Bang Y, Van Custem E, Feyereislova A, et al. Trastuzumab in combination with chemotherapy versus chemotherapy alone for treatment of HER2-positive advanced gastric or gastro-oesophageal junction cancer (ToGA): a phase 3 , open-label, randomised controlled trial. Lancet 2010; 28: 687-97.

https://doi.org/10.1016/S0140-6736(10)61121-X

[5] Kang YK, Chin K, Chung HC, et al. S-1 plus leucovorin and oxaliplatin versus $\mathrm{S}-1$ plus cisplatin as first-line therapy in patients with advanced gastric cancer (SOLAR): a randomized, open-label, phase 3 trial. Lancet Oncol 2020; 21: 1045-56. https://doi.org/10.1016/S1470-2045(20)30315-6

[6] Chihara G, Maeda Y, Hamuro J, Sasaki T, Fukuoka F. Inhibition of mouse sarcoma 180 by polysaccharides from Lentinus edodes (Berk.) Sing. Nature 1969; 222: 687-8. https://doi.org/10.1038/222687a0

[7] Oba K, Kobayashi M, Matsui T, Kodera Y, Sakamoto J. Individual patient based meta-analysis of lentinan for unresectable/recurrent gastric cancer. Anticancer Res 2009; 29: 2739-46.

[8] Wang H, Cai Y, Zheng Y, Bai Q, Xie D, Yu J. Efficacy of biological response modifier lentinan with chemotherapy for advanced cancer: a meta-analysis. Cancer Med 2017; 6: 2222-33.

https://doi.org/10.1002/cam4.1156

[9] Yoshino S, Nishikawa K, Morita S, et al. Randomised phase III study of S-1 alone versus S-1 plus lentinan for unresectable or recurrent gastric cancer (JFMC36-0701). Eur J Cancer 2016; 65: 164-71.

https://doi.org/10.1016/j.ejca.2016.06.012

[10] Chen DS, Mellman I. Oncology meets immunology: the cancer-immunity. Immunity 2013; 39: 1-10.

\section{https://doi.org/10.1016/j.immuni.2013.07.012}

[11] Tumeh PC, Harview CL, Yearley JH, et al. PD-L1 blockade induces responses by inhibiting adaptive immune resistance. Nature 2014; 515: 568-71. https://doi.org/10.1038/nature13954

[12] Ina H, Ina K, Kabeya M, Kayukawa S, Yoshida T, Yoneda M. Lentinan, a Shiitake Mushroom $\beta$-Glucan, Downregulates the Enhanced PD-L1 Expression Induced by Platinum Compounds in Gastric Cancer Cells. J Analytical Oncol 2020 9: 1-10.

[13] Ina K, Furuta R. Image of Month: Complete response of metastatic gastric cancer to chemo-immunotherapy. Indian J Med Res 2017; 146: 141. https://doi.org/10.4103/ijmr.IJMR 13216

[14] Koizumi W, Narahara H, Hara T, et al. S-1 plus cisplatin versus S-1 alone for first line treatment of advanced gastric cancer (SPIRITS trial): A phase III trial. Lancet Oncol 2008; 9: 215-21. https://doi.org/10.1016/S1470-2045(08)70035-4

[15] Hironaka S, Sugimoto N, Yamaguchi K, et al. S-1 plus cisplatin leucovorin versus $\mathrm{S}-1$ plus leucovorin and oxaliplatin versus S-1 plus cisplatin in patients with advanced gastric cancer: a randomized, multicenter, open-label, phase 2 trial. Lancet Oncol 2016; 17: 99-108. https://doi.org/10.1016/S1470-2045(15)00410-6

[16] Japnese Gastric Cancer Association. Japanese gastric cancer treatment guidelines 2014 ver.4. Gastric Cancer 2017; 20: 1-9.

https://doi.org/10.1007/s10120-016-0622-4

[17] Therasse $P$, Arbuck SG, Eisenhauer EA, et al. New guidelines to evaluate the response to treatment in solid tumors. European Organization for research and treatment of cancer, National Cancer Institute of the United States, National Cancer Institute of Canada. J Natl Cancer Inst 2000; 92: 205-16.

https://doi.org/10.1093/jnci/92.3.205 
[18] Kanda $Y$. Investigation of the freely-available easy-to-use software "EZR" (Easy R) for medical statistics. Bone Marrow Transplant 2013; 48: 452-8. https://doi.org/10.1038/bmt.2012.244

[19] Grabosch S, Bulatovic M, Zeng F, et al. Cisplatin-induced immune modulation in ovarian cancer mouse models with distinct inflammation profiles. Oncogene 2019; 38: 2380-93. https://doi.org/10.1038/s41388-018-0581-9

[20] Fournel L, Wu Z, Stadler N, Damotte D, et al. Cisplatin increases PD-L1 expression and optimizes immune checkpoint blockade in non-small lung cancer. Cancer Letters 2019; 464: 5-14. https://doi.org/10.1016/j.canlet.2019.08.005

[21] Aleem E. $\beta$-glucans and their applications in cancer therapy: focus on human studies. Anticancer Agents Med Chem 2013; 13: 709-19.

https://doi.org/10.2174/1871520611313050005
[22] Vetvicka V, Vetvickova J. Anti-infectious and anti-tumor activities of $\beta$-glucans. Anticancer Res 2020; 40: 3139-45. https://doi.org/10.21873/anticanres.14295

[23] Yoshino S, Tahata T, Hazama S, et al. Immunoregulatory effects of the antitumor polysaccharide lentinan on Th1/Th2 balance in patients with digestive cancer. Anticancer Res 2000; 20: 4707-11.

[24] Ina K, Kataoka T, Ando T. The use of lentinan for treating gastric cancer. Anticancer Agents Med Chem 2013; 13: 6818.

https://doi.org/10.2174/1871520611313050002

[25] Ina K, Hirade K, Kabeya M, Kataoka T, Furuta R. Long-term survivors of metastatic gastric cancer for $>5$ years after chemotherapy initiation. Cancer Reports and Reviews 2019; 3: $1-5$.

https://doi.org/10.15761/CRR.1000183

Received on 18-11-2020

Accepted on 15-12-2020

Published on 29-12-2020

DOI: https://doi.org/10.30683/1929-2279.2020.09.07

(C) 2020 Ina et al.; Licensee Neoplasia Research.

This is an open access article licensed under the terms of the Creative Commons Attribution Non-Commercial License (http://creativecommons.org/licenses/by-nc/3.0/) which permits unrestricted, non-commercial use, distribution and reproduction in any medium, provided the work is properly cited. 